\title{
Deprivation influences the emergency admission rate of ambulatory care sensitive conditions
}

\author{
Authors: Richard Conway, ${ }^{\mathrm{A}}$ Deirdre O'Riordan, ${ }^{\mathrm{B}}$ Declan Byrne, ${ }^{\mathrm{C}}$ Sean Cournane, ${ }^{\mathrm{D}}$ Seamus Coveney ${ }^{\mathrm{E}}$ and \\ Bernard Silke ${ }^{\mathrm{F}}$
}

Ambulatory care sensitive conditions (ACSCs) are a group of conditions judged to be suitable for healthcare efficiency initiatives to reduce the rate of hospital admissions. All emergency medical admissions to an Irish hospital between 2002 and 2013 were assessed for ACSCs. They were categorised by quintile of deprivation index and evaluated against hospital admission rate. Univariable and multivariable risk estimates were calculated, using logistic regression or zero-truncated Poisson regression. There were 66,861 admissions in 36,214 patients. ACSCs represented $66.4 \%$ of admissions. The rate of ACSC admissions increased with deprivation index, Q1 10.4 (95\% confidence interval (CI) 10.210.5), Q2 17.3 (95\% CI 17.2-17.5), Q3 34.0 (95\% CI 33.7-34.2), Q4 30.2 (95\% CI 30.0-30.4) and Q5 44.5 (95\% CI 43.8- 45.1) $(p<0.001)$, corresponding incidence rate ratios compared with Q1 were: Q2 1.67 (95\% CI 1.64-1.70), Q3 3.28 (95\% CI 3.22-3.33), Q4 2.92 (95\% CI 2.87-2.97) and Q5 4.29 (95\% CI $4.20-4.39)(p<0.001)$. ACSCs are common in acute medical admissions and are strongly influenced by the underlying social demographics of the population.

KEYWORDS: Deprivation index, emergency medical admission, ambulatory care sensitive condition, admission rate

\section{Introduction}

Increasing demand on health services coupled with spiralling costs has prompted investigation towards maximising healthcare efficiencies. ${ }^{1-5}$ According to the King's Fund, ambulatory care sensitive conditions (ACSC) account for one

Authors: ${ }^{\text {A }}$ specialist registrar, Department of Internal Medicine, St James's Hospital, Dublin, Ireland, and CARD Newman Research Fellow, University College Dublin, Dublin, Ireland; ${ }^{B}$ consultant physician, Department of Internal Medicine, St James's Hospital, Dublin, Ireland; ' Consultant physician, Department of Internal Medicine, St James's Hospital, Dublin, Ireland; 'Dedical physicist, Department of Internal Medicine, St James's Hospital, Dublin, Ireland; Electurer, School of Geographical and Earth Sciences, University of Glasgow, Glasgow, UK; F consultant physician, Department of Internal Medicine, St James's Hospital, Dublin, Ireland in every six emergency hospital admissions in England. ${ }^{6}$ ACSCs are conditions for which effective management and treatment may prevent admission to hospital. They can be classified as: chronic conditions, where effective care can prevent flareups; acute conditions, where early intervention can prevent more serious progression; and preventable conditions, where immunisation and other interventions can prevent illness. ${ }^{7}$ It therefore seems logical to target such conditions as a strategy to avoid unnecessary admissions; it has been stated that high levels of such admissions may indicate poor coordination between the different elements of the healthcare system, and that an emergency admission for an ACSC is a sign of poor overall quality of care. $^{6}$

There are potential limitations to this approach without appropriate reference to the social and age structure of the catchment population. Socioeconomic deprivation may also be relevant to hospital utilisation for ACSCs; ${ }^{6,8}$ a systematic review found that patients from deprived areas attended the emergency department (ED) more frequently, appeared to have attended ED for less serious conditions, and accessed outpatient care more via emergency channels. ${ }^{9}$

In this paper we set out to identify how the deprivation status and age structure of our local population might influence the emergency admission rate for ACSCs, by investigating a database for all emergency medical admissions from 2002 to 2013.

\section{Methods}

\section{Background}

St James' Hospital (SJH) serves as a secondary care centre for emergency admissions for its local Dublin catchment area of 270,000 adults. SJH's catchment area is largely inner city with over $50 \%$ of patients considered to be deprived; hospitals serving deprived catchment areas face particular resource problems in meeting the needs of the population that they serve. ${ }^{10,11}$ Emergency medical patients are admitted from the ED to an acute medical admission unit, the operation and outcome of which have been described elsewhere. ${ }^{4,5}$

\section{Data collection}

For audit purposes we employed an anonymous patient database assembling core information about each clinical episode from elements contained on the patient administration 
system, the national hospital inpatient enquiry (HIPE) scheme, the patient electronic record, the emergency room and laboratory systems. HIPE is a national database of coded discharge summaries from acute public hospitals in Ireland. ${ }^{12,13}$ Ireland used the International classification of diseases, 9th revision, clinical modification (ICD-9-CM) for both diagnosis and procedure coding from 1990 to 2005, and ICD-10-CM since then.

Data held on the database include the unique hospital number, admitting consultant, date of birth, gender, area of residence, principal and up to nine additional secondary diagnoses, principal and up to nine additional secondary procedures, and admission and discharge dates. Additional information cross-linked and automatically uploaded to the database includes physiological, haematological and biochemical parameters. For the present study data were related to all emergency general medical patients admitted to $\mathrm{SJH}$ from 2002 to 2013.

Approximately $11.5 \%$ of our patients stay $>30$ days with a median length of stay (LOS) of 55.0 days (inter-quartile range (IQR) 38.8-97.6) - many of these are awaiting nursing home beds or other social interventions rather than ongoing admissions for medical reasons. We have therefore chosen a truncated end-point (death or episode completed by the 30-day endpoint) for analysis, to avoid the additional confounding of non-medical reasons for continuing as an inpatient.

\section{ACSCS}

Discharge codes were interrogated to construct the total occurrence as well as the number of such ACS diagnoses present per patient. These codes were broadly by system: i) cardiovascular - angina, essential hypertension, congestive heart failure; ii) respiratory - chronic obstructive airways disease, asthma, pneumonia/influenza; iii) neurological convulsions/epilepsy; iv) gastrointestinal - gastrointestinal bleeding/perforated ulcer, gastroenteritis/dehydration; v) diabetes; vi) renal - pyelonephritis; vii) infective cellulitis, ENT infections, pelvic inflammatory diease; viii) haematological - anaemia; ix) nutritional deficiencies; $\mathrm{x}$ ) gangrene; and xi) dental conditions. We categorised patients into five groups on this basis, zero meaning no ACSC present. As only $2.7 \%$ of patients had four or more, we grouped all patients with four or more ACSC present into the top category.

\section{Deprivation indices}

To classify deprivation, we used the public domain Irish National Deprivation Index for Health and Health Services Research, derived by the Small Area Health Research Unit at Trinity College Dublin. ${ }^{14}$ We have described the relevant methodology in more detail previously. ${ }^{11}$ From the 2006 census returns, information by electoral division was available in respect of the total population within each unit area and the age dependency ratio (proportion of population $<15$ years or $>64$ years old). The incidence rate (rate/1,000 population) for emergency medical admissions for each electoral division was calculated by the average yearly admissions divided by the total population.

\section{Statistical methods}

Descriptive statistics were calculated for background demographic data, including mean/standard deviation (SD), median/ IQR or percentages. Comparisons between categorical variables and mortality were made using chisquare tests. We used a stepwise logistic regression model to predict 30-day in-hospital death, adjusting risk estimates for major predictor variables. We used margins to estimate and interpret adjusted predictions for sub-groups, while controlling for other variables, using computations of average marginal effects. In the multivariate model, we adjusted for previously determined outcome predictor variables of acute illness severity, ${ }^{15,16}$ Charlson comorbidity index, ${ }^{17}$ sepsis status ${ }^{18}$ and chronic disabling disease score. ${ }^{19}$ For LOS data, we employed a zero-truncated Poisson regression model including predictive categorical variables in the model as a series of indicator variables. We used robust standard errors for the parameter estimates. ${ }^{20}$ The Poisson regression coefficients are the log of the rate ratio, the rate at which events occur is called the incidence rate. Thus we can interpret the coefficients in terms of incidence rate ratios (IRRs).

Adjusted odds ratios (OR) and 95\% confidence intervals (CI) or IRRs were calculated for those predictors that significantly entered the multivariate model. Statistical significance at $\mathrm{p}<0.05$ was assumed throughout. Stata version 13.1 (Stata Corp, College Station, TX, USA) statistical software was used for analysis.

Table 1. Emergency admissions (2002-2013) by ACSC (present or absent).

\begin{tabular}{lllll} 
Factor & Level & Non-ACSC & ACSC & p value \\
Emergency admissions, $n$ & & 22,495 & 44,438 & $<0.001$ \\
Gender, $\mathrm{n}(\%)$ & Male & $9,512(42.3)$ & $23,221(52.3)$ & $<1,217(47.7)$ \\
& Female & $12,983(57.7)$ & $41,934(94.4)$ & $<0.001$ \\
Outcome & Alive & $21,839(97.1)$ & $2,504(5.6)$ & $<0.001$ \\
& Died & $656(2.9)$ & $67.2(48.9-78.8)$ & $<0.001$ \\
Age, median (IQR) & & $49.2(33.1-69.9)$ & $5.8(2.7-10.7)$ & $<$ \\
LOS, days (IQR) & $3.8(1.2-8.0)$ & & \\
\hline
\end{tabular}

$\mathrm{ACSC}=$ ambulatory care sensitive conditions; IQR = inter-quartile range; $\mathrm{LOS}=$ length of stay. 


\begin{tabular}{|c|c|c|c|c|}
\hline Parameter & Group & IRR & $95 \% \mathrm{CI}$ & $p$ value \\
\hline \multirow[t]{4}{*}{ Age dependency } & QI vs QII & 1.31 & $1.23-1.40$ & $<0.001$ \\
\hline & QI vs QIII & 2.06 & $1.93-2.20$ & $<0.001$ \\
\hline & QI vs QIV & 1.78 & $1.68-1.88$ & $<0.001$ \\
\hline & QI vs QV & 1.96 & $1.87-2.06$ & $<0.001$ \\
\hline \multirow[t]{4}{*}{ Deprivation quintile } & QI vs QII & 2.04 & $1.94-2.15$ & $<0.001$ \\
\hline & QI vs QIII & 3.42 & $3.23-3.61$ & $<0.001$ \\
\hline & QI vs QIV & 3.47 & $3.32-3.64$ & $<0.001$ \\
\hline & QI vs QV & 4.60 & $4.33-4.88$ & $<0.001$ \\
\hline
\end{tabular}

\section{Results}

\section{Patient demographics}

A total of 66,861 admissions were recorded in 36,214 patients over the 12-year study period (2002-2013). These represented all emergency general medical admissions. The proportion of males and females were 48.9 and $51.1 \%$ respectively. The median (IQR) LOS was 5.1 (2.1-9.8) days. The median (IQR) age was $62.2(42.0-77.1)$ years, with the upper $10 \%$ boundary at 84.5 years.
The major disease categories were respiratory $(26.0 \%)$, cardiovascular $(16.5 \%)$, neurological $(16.2 \%)$, gastrointestinal (10.7\%), hepatobilary (5.0\%) and renal (4.4\%). The Charlson comorbidity score of 0,1 , or 2 was present in 45.7, 27.3 and $27.0 \%$ respectively. The frequency of no blood culture, negative blood culture, and positive blood culture was 75.4, 21.2 and $3.4 \%$ of patients, with respective 30 -day mortality rates of 5.5, 16.2 and $31.8 \%$.

\section{Demographics of ACSCs}

The demographic characteristics of patients with and without ACSCs are shown in Table 1. Males are over-represented for ACSC (52.3 vs 47.7\%; $\mathrm{p}<0.001)$. The median age of ACSC was 18 years older (67.2 (IQR 48.9-78.8) vs 49.2 years (IQR 33.1-69.9); $\mathrm{p}<0.001)$. The in-hospital mortality was higher (5.6 vs 2.9\%; $\mathrm{p}<0.001$ ) and LOS was longer (5.8 (IQR 2.7-10.7) vs 3.8 days (IQR $1.2-8.0$ ); $\mathrm{p}<0.001$ ). Patients with ACSCs had higher levels of acute illness severity, Charlson comorbidity and chronic disabling disease.

\section{Deprivation quintile and hospital admissions with ACSCs}

We examined the rate of admissions, for patients with ACSC, by their deprivation status (Figs 1 and 2). The admission rates per 1,000 population for ACSC patients by deprivation quintile were Q1 10.4 (95\% CI 10.2-10.5), Q2 17.3 (95\% CI 17.2-17.5), Q3 34.0 (95\% CI 33.7-34.2), Q4 30.2 (95\% CI $30.0-30.4)$ and Q5 44.5 (95\% CI 43.8-45.1) ( $<<0.001$ for each

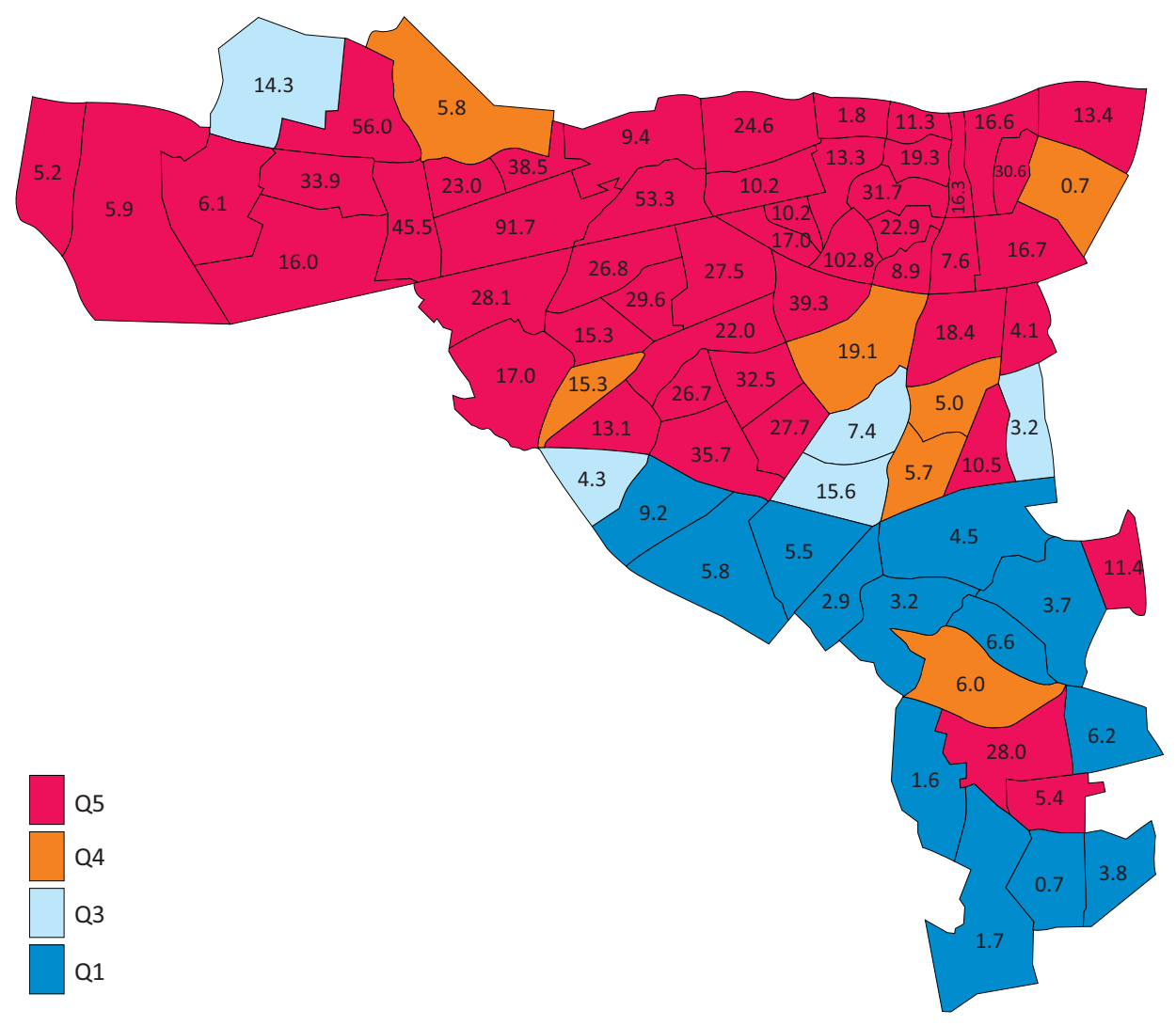

Fig 1. Spatial distribution (by electoral division) of incidence admission rates for ACSC of patients resident in the St James' Hospital catchment area. Their median admission incidence rate was 27.7 (16.4-34.7) with a range of $0.7-102.8 / 1,000$ population. ACSC $=$ ambulatory care sensitive conditions. 


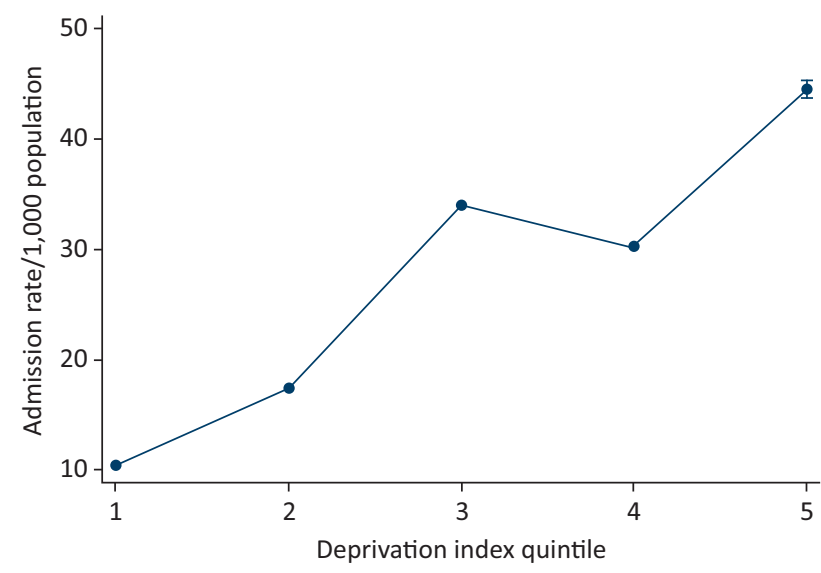

Fig 2. Relationship between deprivation score and the admission incidence (rate/1,000 population) for ACSC. The predicted probabilities were derived from the multivariable truncated Poisson model using Stata's margins command; the effect with confidence interval is plotted based on the latter prediction. $\mathrm{ACSC}=$ ambulatory care sensitive conditions.

rate). The IRRs by deprivation quintile were (compared with Q1): Q2 1.67 (95\% CI 1.64-1.70), Q3 3.28 (95\% CI 3.22-3.33), Q4 2.92 (95\% CI 2.87-2.97) and Q5 4.29 (95\% CI 4.20-4.39) $(\mathrm{p}<0.001$ for each).

The significance of an ACSC diagnosis in terms of outcome (30-day in-hospital mortality and LOS)

ACSCs were present in 44,438 admissions (66.4\%); 35.5, 20.3, 8.0 and $2.7 \%$ of admissions had ACSC scores of $1,2,3$, and $4+$ respectively. Overall these conditions were associated with an increased risk of in-hospital mortality (OR 1.79; 95\% CI 1.62-1.98). The mortality increased with the number of ACSCs present; the respective rates were 4.5, 8.2, 14.3, 20.4 and $27.6 \%(\mathrm{p}<0.001)$ with $1,2,3$, and $4+$ such diagnoses (Fig 3 ). The ORs for 30-day in-hospital mortality for the number of

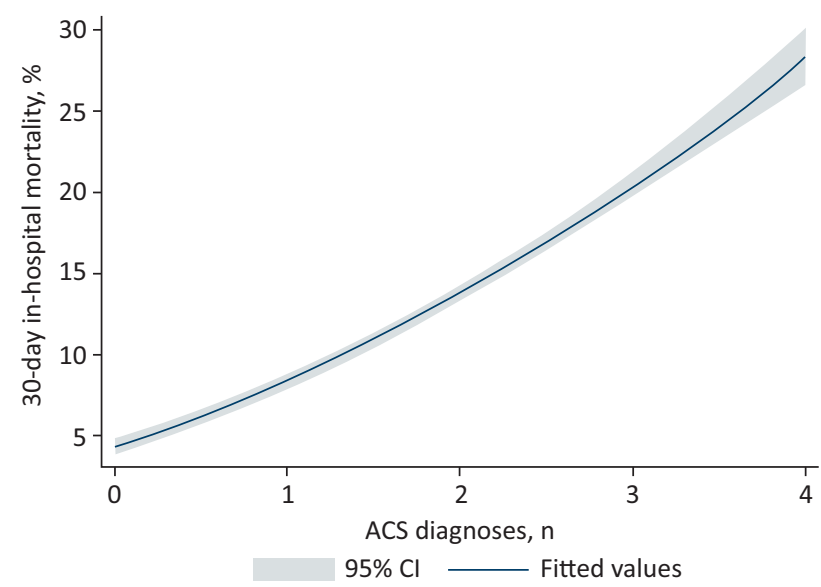

Fig 3. Relationship between ACSC (number per patient) and 30-day inhospital mortality. The individual mortality figures at each level have been fitted to a quadratic model with the estimated confidence intervals. ACSC $=$ ambulatory care sensitive conditions.
ACSC diagnoses (compared with none) were 1.39 (95\% CI 1.24-1.56), 2.00 (95\% CI 1.78-2.25), 2.49 (95\% CI 2.16-2.87) and 2.97 (95\% CI 2.45-3.61) ( $\mathrm{p}<0.001$ for each comparison). However, ACSCs were associated with more acute illness severity, Charlson comorbidity, and chronic disabling disease. Adjustment for such factors indicated that the increased mortality was due to these associated factors, rather than an intrinsic relationship with the ACSCs - fully adjusted OR 0.71 (95\% CI 0.63-0.79).

The data suggested that ACSC patients required a significantly longer LOS with an IRR of 1.28 (95\% CI 1.26-1.31) $(\mathrm{p}<0.001)$ With increasing complexity, as evidenced by the number of such diagnoses (1, 2, 3 and 4+ ACSC), the IRR's respectively were 1.18 (95\% CI 1.15-1.20), 1.33 (95\% CI 1.30- 1.36), 1.48 (95\% CI 1.43-1.52) and 1.59 (95\% CI 1.52-1.65) $(\mathrm{p}<0.001$ for each comparison). This was due to the associated disease acuity and complexity; adjusted for these factors such as acute illness severity, Charlson comorbidity, and chronic disabling disease the LOS was in fact slightly lower - IRR 0.97 (95\% CI 0.96-0.98).

\section{Discussion}

This study shows that ACSCs are common in our cohort with two-thirds of emergency medical admissions having at least one. The coexistence of multiple ACSCs was common, with one-third and one-fifth of patients having two and three ACSCs respectively. While the high frequency of these intervention amenable conditions would seem promising, our other findings are more sobering in this regard. Patients with ACSCs were significantly older, more deprived, and had greater illness severity and comorbidity burdens. This does not exclude the merit of targeting these conditions; however we must be pragmatic in our expectations with regard to this frail elderly group of patients.

The variation in the admission rates of ACSCs in the UK has often been suggested to be a reflection of differences in the quality and integration of care. A variation in the degree of deprivation present in, and indeed within, the catchment areas of institutions provides an alternative explanation. In our study apparent differences in admission rates due to ACSCs were explained by differences in deprivation. Our data add to the literature in this area by showing differences in admission rates for ACSCs by areas of differing deprivation to one central hospital, excluding centre-specific factors as an explanation. Investment in improving socioeconomic conditions within the population may be a more effective method of achieving benefits in outcomes for these conditions.

Our study has a number of limitations. This was a single centre study and results may not be generalisable to the healthcare system at large, particularly in areas serving differing socioeconomic profiles. The external validity of our results requires confirmation in other populations and studies. Our results are limited to general medical patients as for reasons related to data collection we have not evaluated patients admitted to other services. The diagnosis of an ACSC was based on a discharge rather than admission diagnosis for practical reasons, for a significant proportion of admissions the final diagnosis is not confirmed at the time of admission.

In conclusion our study shows ACSCs to be common. Patients with ACSCs are on average older and sicker. Deprivation rather 
than ACSC burden alone explained increased rates of admission for those with such conditions.

\section{References}

1 Litvak E, Bisognano M. More patients, less payment: increasing hospital efficiency in the aftermath of health reform. Health Aff (Millwood) 2011;30:76-80.

2 Moses HI, Matheson D, Dorsey E et al. The anatomy of health care in the United States. JAMA 2013;18:1947-63.

3 Curfman G, Morrissey S, Drazen J. High-value health care - a sustainable proposition. N Engl J Med 2013;369:1163-4.

4 Rooney T, Moloney ED, Bennett K et al. Impact of an acute medical admission unit on hospital mortality: a 5-year prospective study. QJM 2008;101:457-65.

5 Conway R, O'Riordan D, Silke B. Long-term outcome of an AMAU a decade's experience. QJM 2014;107:43-9.

6 Tian Y, Dixon A, Gao H. Emergency hospital admissions for ambulatory care-sensitive conditions: identifying the potential for reductions. London: The King's Fund, 2012.

7 Ham C, Imison C, Jennings M. Avoiding hospital admissions: lessons from evidence and experience. London: The King's Fund, 2010.

8 Zhao Y, You J, Guthridge SL, Lee AH. A multilevel analysis on the relationship between neighbourhood poverty and public hospital utilization: is the high indigenous morbidity avoidable? BMC Public Health 2011;11:737.

9 McCormick B, Hill P-S, Emmi P. Are hospital services used differently in deprived areas? Evidence to identify commissioning challenges. Oxford: Centre for Health Service Economics and Organisation, 2012.

10 Walsh JB, Coakley D, Murphy C et al. Demographic profile of the elderly population in Dublin accident and emergency hospital catchment areas. Ir Med J 2004;97:84-6.
11 Conway R, Galvin S, Coveney S et al. Deprivation as an outcome determinant in emergency medical admissions. QJM 2013;106:245-51.

12 O'Loughlin R, Allwright S, Barry J et al. Using HIPE data as a research and planning tool: limitations and opportunities. Ir J Med Sci 2005;174:40-5; discussion 52-7.

13 O'Callaghan A, Colgan MP, McGuigan C et al. A critical evaluation of HIPE data. Ir Med J 2012;105:21-3.

14 Kelly A, Teljeur C. SAHRU National Deprivation Index. Dublin: Trinity College, 2007.

15 Silke B, Kellett J, Rooney T et al. An improved medical admissions risk system using multivariable fractional polynomial logistic regression modelling. QJM 2010;103:23-32.

16 O'Sullivan E, Callely E, O'Riordan D et al. Predicting outcomes in emergency medical admissions - role of laboratory data and comorbidity. Acute Med 2012;2:59-65.

17 Charlson ME, Pompei P, Ales KL et al. A new method of classifying prognostic comorbidity in longitudinal studies: development and validation. J Chronic Dis 1987;40:373-83.

18 Chotirmall SH, Callaly E, Lyons J et al. Blood cultures in emergency medical admissions: a key patient cohort. Eur J Emerg Med 2014, epub ahead of print.

19 Chotirmall SH, Picardo S, Lyons J et al. Disabling disease codes predict worse outcomes for acute medical admissions. Intern Med J 2014;44:546-53.

20 Cameron A, Trivedi P. Microeconometrics using stata. College Station, TX: Stata Press, 2009.

Address for correspondence: Dr B Silke, Department of Internal Medicine, St James's Hospital, Dublin 8, Ireland. Email: bernardsilke@physicians.ie

New to systematic reviewing, or need to improve your critical appraisal skills?

Our intensive 1-day courses will introduce you to the key principles of critical appraisal and systematic reviewing, and equip you with the basic skills to put your knowledge into practice.

> Introduction to critical appraisal

> Systematic reviews and meta-analysis in action

Discount available if both courses are booked together.

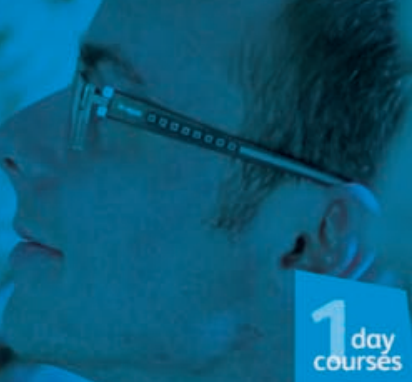

'Informative and practical'

Dr Rajeswari Ramaraj

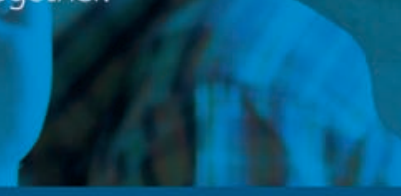

